Pacific Journal of Mathematic 


\title{
COMPLETELY MONOTONIC FUNCTIONS ON CONES
}

\author{
WAlter M. Gilbert
}

1. Introduction. A function $f(x), 0 \leqq x<\infty$, is said to be completely monotonic on $0 \leqq x<\infty$ if $(-1)^{n} f^{(n)}(x) \geqq 0$ for $0<x<\infty$ and $f(0)=f(0+)$. A similar and equivalent definition involving differences is available. A fundamental theorem regarding such functions, proved (independently) by Hausdorff, Bernstein and Widder, states that they are the class of Laplace-Stieltjes transforms of bounded monotone functions. Several of the many known proofs are given in Widder [3], which also gives references for other proofs. The corresponding theorem for two dimensions has been proved by Schoenberg [2]. It is not difficult to construct a proof for $n$-dimensions along the lines of the original proof of Hausdorff and in the process establish the equivalence of the corresponding derivative and difference criteria.

In this note we wish to introduce a class of functions, defined on $n$-dimensional polyhedral cones with vertex at the origin, which we call completely monotonic $(A)$, and, in analogy with the theorem of Hausdorff-Bernstein-Widder, show that they are the Laplace-Stieltjes transforms of bounded monotone functions on the "conjugate space" $t=\left(t_{1}\right.$, $\left.\cdots, t_{n}\right)$ with $\sum_{i=1}^{n} x_{i} t_{i} \geqq 0$. We then show that a function completely monotonic $(A)$ on each of a set of overlapping cones may be represented by a single integral, which may then be used to extend the function to the convex closure of the set of cones. Lastly, we show by an example that a function may be completely monotonic along every line with nonnegative slope in the first quadrant without being completely monotonic as a function of two variables.

2. Functions completely monotonic on cones. We commence with some notations and definitions. We shall write $x$ in place of $\left(x_{1}, \cdots, x_{n}\right)$, $x t^{\prime}$ in place of $\left(x_{1} t_{1}+\cdots+x_{n} t_{n}\right)$, and where these appear in integrands we shall use a single integral sign to denote a multiple integral.

For a given convex cone $D, D^{*}$ will be the set of all $t$ such that $\sum_{i=1}^{n} x_{i} t_{i} \geqq 0$ for all $x$ in $D$. By an $n$-cone we shall mean a convex cone in $E_{n}$ spanned by $n$ linearly independent vectors $x^{i}=\left(x_{1}^{i}, \cdots, x_{n}^{i}\right)$, and such that there is a hyperplane having only the origin in common with

Received August 19, 1954, and in revised form June 24, 1955. Part of this paper is a part of a dissertation submitted to Princeton University. We wish to acknowledge our gratitude to Professor S. Bochner under whose direction the dissertation was prepared. We are also grateful to the referee for several valuable suggestions. 
the cone. We shall say that $\left\{D_{\sigma}\right\}, \sigma \in S$, is a collection of overlapping $n$-cones if it is impossible to divide the index set $S$ into subsets $S^{\prime}$ and $S^{\prime \prime}, S=S^{\prime} \cup S^{\prime \prime}$, so that $\bigcup_{S^{\prime}} D_{\sigma}$ and $\bigcup_{S^{\prime \prime}} D_{\sigma}$ as point sets in $E_{n}$ have only the origin in common.

Let $f(x)$ be defined on an $n$-cone $D$ and be continuous on the boundary. Then $f(x)$ will be said to be completely monotonic $(A)$ on $D$ if

$$
\sum_{i_{1}=0}^{m_{1}} \cdots \sum_{i_{n}=0}^{m_{n}}\left(\begin{array}{c}
m_{1} \\
i_{1}
\end{array}\right) \cdots\left(\begin{array}{c}
m_{n} \\
i_{n}
\end{array}\right)(-1)^{i_{1}+\cdots+i_{n}} f\left(x+i_{1} \delta_{1} x^{1}+\cdots+i_{n} \delta_{n} x^{n}\right) \geqq 0
$$

for any $x$ in $D$ and any $\delta_{i} \geq 0$. If $D$ is the positive orthant, a function completely monotonic $(A)$ on $D$ is completely monotonic in the ordinary sense.

For reference purposes we now state the ordinary form of the Hausdorff-Bernstein-Widder Theorem for several variables. A proof paralleling the proof given for one dimension in Widder [3], p. 162, is not difficult.

THeOREM 2.1. A necessary and sufficient condition that $f(x)$ be completely monotonic on $0 \leqq x_{i}<\infty, i=1,2, \cdots, n$, is that

$$
f(x)=\int_{0}^{\infty} e^{-x t^{\prime}} d \varphi(t),
$$

where $\varphi(t)$ is bounded and monotone (in the sense of [1]) and the integral is convergent for $0 \leqq x_{i}, i=1,2, \cdots, n . \varphi(t)$ is essentially unique.

From this we proceed to the corresponding theorem for $n$-cones.

THEOREM 2.2. Let $D$ be an n-cone. Then a necessary and sufficient condition that a function $f(x)$ be completely monotonic $(A)$ on $D$ is that

$$
f(x)=\int_{D^{*}} e^{-x t^{\prime}} d \varphi(t),
$$

where $\varphi(t)$ is bounded and monotone in $D^{*}$, and the integral is convergent for $x$ in $D . \varphi(t)$ is essentially unique.

Proof. Suppose that $f(x)$ is completely monotonic $(A)$ on an $n$-cone $D$. Let $T$ be a linear transformation which carries $D$ into the positive orthant $P$. Let $g(x)=f\left[T^{-1}(x)\right]$. Then $g(x)$ is completely monotonic, since the differences taken along lines parallel to the edges of the cone are transformed into differences taken parallel to the axes. Then

$$
g(x)=\int_{0}^{\infty} e^{-x t^{\prime}} d \psi(t)
$$


where $\psi$ is bounded and monotone, by Theorem 2.1. Let $U$ be the linear transformation on $t$ such that $\sum_{i=1}^{n} x_{i} t_{i}=\sum_{i=1}^{n} x_{i}^{\circ} t_{i}^{\circ}$, where $x^{\circ}=T(x)$ and $t^{\circ}=U(t)$. For any set $S$ in the $t$ domain let $\varphi(S)=\psi\left[U^{-1}(S)\right] . \quad \varphi(t)$ is clearly monotone and so (I) holds.

Suppose, on the other hand, that we have (I) with $\varphi(t)$ bounded and monotone in $D^{*}$. We use a linear transformation $T$ to carry $D$ onto the positive orthant and a $U$, defined as above, to carry $D^{*}$ onto $P^{*}$. We then have a function $g(x)$ defined on $P$ and equal there to

$$
\int_{0}^{\infty} e^{-x t^{\prime}} d \psi(t)
$$

for a bounded monotone $\psi(t)$. The function $g(x)$ is thus completely monotonic, from Theorem 2.1, and this property will carry over into complete monotonicity $(A)$ for $f(x)$ when we apply $T^{-1}$. This completes the proof of the theorem.

We now consider functions which are completely monotonic $(A)$ on each of a collection of overlapping $n$-cones.

THEOREM 2.3. Suppose that $f(x)$ is completely monotonic $(A)$ on each of a collection $\left\{D_{\sigma}\right\}, \sigma \in S$, of overlapping n-cones. Suppose also that if the intersection of any two cones in $\left\{D_{\sigma}\right\}$ contains a point other than the origin it contains an open set. Then all of the $\varphi_{\sigma}(t)$ as defined in Theorem 2.2 are equal, and are zero outside $\left(\bigcup_{S} D_{\sigma}\right)^{*}$.

Proof. We note that always $\left(\cup D_{\sigma}\right)^{*}=\cap D_{\sigma}^{*}$. To begin with, suppose that $D$ consists of two cones, $D_{1}$ and $D_{2}$. Consider a point $x$ in their intersection. From Theorem 2.2

$$
f(x)=\int_{D_{1}^{*}} e^{-x t^{\prime}} d \varphi_{1}(t) .
$$

This may be extended to an integral over $D_{1}^{*} \cup D_{2}^{*}$ by defining $\varphi_{1}(t)=0$ for $t$ in $D_{2}^{*}-D_{1}^{*}$. At the same time

$$
f(x)=\int_{D_{2}^{*}} e^{-x t^{\prime}} d \varphi_{2}(t),
$$

and $\varphi_{2}(t)$ can likewise be defined to be zero outside $D_{1}^{*}$. Since both of these representations are valid in an $n$-cone contained in $D_{1} \cap D_{2}, \varphi_{1}(t)=$ $\varphi_{2}(t)$ by the uniqueness condition.

Consider the general case, and suppose the theorem to be false. Then there are two cones, $D_{1}$ and $D_{2}$, say, such that $\varphi_{2}(t)$ is not zero somewhere outside $D_{1}^{*}$. Let the collection of cones $\left\{D_{\sigma}\right\}, \sigma \in S^{\prime}$, be those for which $\varphi_{\sigma}$ is zero outside $D_{1}^{*}$; let the other cones form $\left\{D_{\sigma}\right\}, \sigma \in S^{\prime \prime}$. 
Neither $S^{\prime \prime}$ nor $S^{\prime \prime}$ is empty, $S^{\prime} \cup S^{\prime \prime}=S$, and $S^{\prime} \cap S^{\prime \prime}=\phi$. Thus $\bigcup_{S^{\prime}} D_{\sigma}$ and $\bigcup_{S^{\prime \prime}} D_{\sigma}$ have a point other than the origin in common, and there is a $D_{\sigma^{\prime}}$, $\sigma^{\prime} \in S^{\prime}$, and a $D_{\sigma^{\prime \prime}}, \sigma^{\prime \prime} \in S^{\prime \prime}$, whose intersection contains an open set. By the first part of the theorem $\varphi_{\sigma^{\prime}}(t)=\varphi_{\sigma^{\prime \prime}}(t)$, and this is a contradiction.

It is known that $D^{* *}$ is the convex closure of $D$, where $D$ is any (possibly non-convex, non-polyhedral) cone. Also,

$$
\int_{D^{*}} e^{-x t^{\prime}} d \varphi(t) \leqq \int_{D^{*}} d \varphi(t)
$$

for any $x$ in $D^{* *}$. Thus we may use the integral representation to extend a function of the sort described in Theorem 2.3 to the convex closure of $\bigcup_{S} D_{\sigma}$. We state this as a corollary.

CoRollary. Suppose $f(x)$ is completely monotonic $(A)$ on each of a set $\left\{D_{\sigma}\right\}$ of n-cones as in Theorem 2.3. Then $f(x)$ may be continued to the convex closure $K$ of $\bigcup_{S} D_{\sigma}$ in such a fashion that it will be completely monotonic $(A)$ on any $n$-cone in $K$.

3. Functions completely monotonic on lines. Using Theorem 2.3 we can deduce complete monotonicity $(A)$ on large cones from complete monotonicity $(A)$ on small cones. The conclusion is false, however, if the small cones are replaced by lines. In fact we can exhibit a function completely monotonic along any line with suitable slope, through the origin or not, which fails to be completely monotonic in several variables. For the sake of simplicity we will discuss an example in two dimensions. Consider the function

$$
\varphi\left(t_{1}, t_{2}\right)=\left\{\begin{array}{l}
1 \text { for }\left(0 \leqq t_{1} \leqq 3,0 \leqq t_{2} \leqq 3\right) \text { except for }\left(1 \leqq t_{1} \leqq 2,1 \leqq t_{2} \leqq 2\right) \\
-1 \text { for }\left(1 \leqq t_{1} \leqq 2,1 \leqq t_{2} \leqq 2\right) \\
0 \text { otherwise. }
\end{array}\right.
$$

Let

$$
f\left(x_{1}, x_{2}\right)=\int_{0}^{\infty} \int_{0}^{\infty} e^{-t_{1} x_{1}-t_{2} x_{2}} \varphi\left(t_{1}, t_{2}\right) d t_{1} d t_{2} .
$$

By Theorem $2.1 f\left(x_{1}, x_{2}\right)$ cannot be completely monotonic. Let

$$
g_{\theta}\left(x_{1}^{\prime}, x_{2}^{\prime}\right)=f\left(x_{1}^{\prime} \cos \theta-x_{2}^{\prime} \sin \theta, x_{2}^{\prime} \cos \theta+x_{1}^{\prime} \sin \theta\right) ;
$$

that is, rotate the axes through an angle $\theta$. We will now show that $g_{\theta}\left(x_{1}^{\prime}, x_{2}^{\prime}\right)$ is completely monotonic on $0 \leqq x_{1}^{\prime}<\infty$ for any fixed value of $x_{2}^{\prime}$ and any $0 \leqq \theta \leqq \pi / 2$. To this end let

$$
u_{1}=t_{1} \cos \theta+t_{2} \sin \theta \text { and } u_{2}=-t_{1} \sin \theta+t_{2} \cos \theta \text {, }
$$


so that

$$
g_{\theta}\left(x_{1}^{\prime}, x_{2}^{\prime}\right)=\iint e^{-x_{1}^{\prime} u_{1}-x_{2}^{\prime} u_{2}} \psi\left(u_{1}, u_{2}\right) d u_{1} d u_{2},
$$

where $\psi\left(u_{1}, u_{2}\right)$ is zero outside a rotated square. We can replace the multiple integral by a repeated integral:

$$
g_{\theta}\left(x_{1}^{\prime}, x_{2}^{\prime}\right)=\int_{0}^{3 \cos \theta+3 \sin \theta} e^{-x_{1}^{\prime} u_{1}} d u_{1} \int e^{-x_{2}^{\prime} u_{2}} \psi\left(u_{1}, u_{2}\right) d u_{2} .
$$

Now the inner integral will always be positive, because any line which intersects the square on which $\psi$ is not zero will have a greater length in the positive region than in the negative, and can thus make only a positive contribution to the integral. Since the inner integral is positive, $g_{\theta}\left(x_{1}^{\prime}, x_{2}^{\prime}\right)$ must be completely monotonic from the one dimensional version of Theorem 2.1.

If $\theta$ is allowed outside the interval $0 \leqq \theta \leqq \pi / 2$, the inner integral will remain positive but the range of the outer integral will extend outside $0 \leqq u_{1}<\infty$. The function $g_{\theta}(a+b, c+d)$ will then be a "kernel of positive type" in $a$ and $b$ for fixed $c$ and $d$ (and vice versa), as discussed in Chapter VI, $\S \S 20-21$ of Widder [3]. If the square upon which $\varphi\left(t_{1}, t_{2}\right)$ is nonzero is moved sufficiently far from the origin along the line $t_{1}=t_{2}$ the corresponding $g_{\theta}\left(x_{1}^{\prime}, x_{2}^{\prime}\right)$ may be made completely monotonic for $0 \leqq x_{1}^{\prime}<\infty$ for $-\pi / 4+\delta \leqq \theta \leqq 3 \pi / 4-\delta$ for any small positive $\delta$ and any fixed $x_{2}^{\prime}$.

\section{REFERENCES}

1. S. Bochner, Monotone Funktionen, Stieltjessche Integrale und harmonische Analyse, Math. Ann., 108 (1933), 378-410.

2. I. J. Schoenberg, Systems of linear inequalities, Trans. Amer. Math. Soc., 35 (1933), $452-478$.

3. D. V. Widder, The Laplace transform, Princeton, 1941.

State College of Washington 



\section{PACIFIC JOURNAL OF MATHEMATICS}

\section{EDITORS}

H. L. Royden

Stanford University

Stanford, California

E. Hewits

University of Washington

Seattle 5 , Washington
R. P. Dilworth

California Institute of Technology Pasadena 4, California

E. G. Straus

University of California

Los Angeles 24, California

\section{ASSOCIATE EDITORS}
E. F. BECKENBACH
C. E. BURGESS
H. BUSEMANN
H. FEDERER

\author{
M. HALL \\ P. R. HALMOS \\ V. GANAPATHY IYER \\ R. D. JAMES
}

\author{
M. S. KNEBELMAN \\ I. NIVEN \\ T. G. OSTROM \\ M. M. SCHIFFER
}

\section{J. J. STOKER \\ G. SZEKERES \\ F. WOLF \\ K. YOSIDA}

\section{SUPPORTING INSTITUTIONS}

UNIVERSITY OF BRITISH COLUMBIA CALIFORNIA INSTITUTE OF TECHNOLOGY

UNIVERSITY OF CALIFORNIA

MONTANA STATE UNIVERSITY

UNIVERSITY OF NEVADA

OREGON STATE COLLEGE

UNIVERSITY OF OREGON

UNIVERSITY OF SOUTHERN CALIFORNIA
STANFORD UNIVERSITY

UNIVERSITY OF UTAH

WASHINGTON STATE COLLEGE

UNIVERSITY OF WASHINGTON

$*$ * * *

AMERICAN MATHEMATICAL SOCIETY

CALIFORNIA RESEARCH CORPORATION HUGHES AIRCRAFT COMPANY

Mathematical papers intended for publication in the Pacific Journal of Mathematics should be typewritten (double spaced), and the author should keep a complete copy. Manuscripts may be sent to any of the editors. Manuscripts intended for the outgoing editors should be sent to their successors. All other communications to the editors should be addressed to the managing editor, E. G. Straus at the University of California, Los Angeles 24, California.

50 reprints of each article are furnished free of charge; additional copies may be obtained at cost in multiples of 50 .

The Pacific Journal of Mathematics is published quarterly, in March, June, September, and December. The price per volume (4 numbers) is $\$ 12.00$; single issues, $\$ 3.50$. Back numbers are available. Special price to individual faculty members of supporting institutions and to individual members of the American Mathematical Society: $\$ 4.00$ per volume; single issues, $\$ 1.25$.

Subscriptions, orders for back numbers, and changes of address should be sent to Pacific Journal of Mathematics, 2120 Oxford Street, Berkeley 4, California.

Printed at Kokusai Bunken Insatsusha (International Academic Printing Co., Ltd.), No. 10, 1-chome, Fujimi-cho, Chiyoda-ku, Tokyo, Japan.

\section{PUBLISHED BY PACIFIC JOURNAL OF MATHEMATICS, A NON-PROFIT CORPORATION}

The Supporting Institutions listed above contribute to the cost of publication of this Journal, but they are not owners or publishers and have no responsibility for its content or policies. 


\section{Pacific Journal of Mathematics}

\section{Vol. 6, No. 4}

1956

Seymour Ginsburg, On mappings from the family of well ordered subsets of

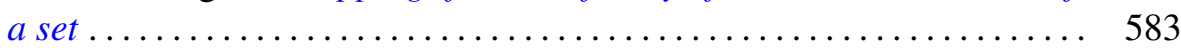

Leon Ehrenpreis, Some properties of distributions on Lie groups ......... 591

Marion K. Fort, Jr., A geometric problem of Sherman Stein ............. 607

Paul R. Garabedian, Calculation of axially symmetric cavities and jets . . . . 611

Walter Mossman Gilbert, Completely monotonic functions on cones ...... 685

William L. Hart and T. S. Motzkin, A composite Newton-Raphson gradient method for the solution of systems of equations ................. 691

C. W. Mendel and I. A. Barnett, A functional independence theorem for square matrices ................................. 709

Howard Ashley Osborn, The problem of continuous programs .......... 721

William T. Reid, Oscillation criteria for linear differential systems with complex coefficients ............................. 733

Irma Reiner, On the two-adic density of representations by quadratic

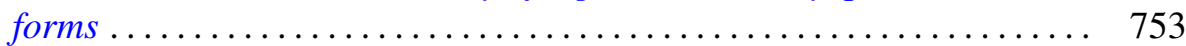

Shoichiro Sakai, A characterization of $W^{*}$-algebras .............. 763

Robert Steinberg, Note on a theorem of Hadwiger................. 775

$\mathrm{J}$. Eldon Whitesitt, Construction of the lattice of complemented ideals within

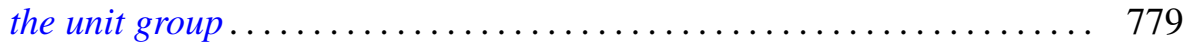

Paul Civin, Correction to "Some ergodic theorems involving two operators"... 